\title{
Comparação de dois métodos de avaliação da fenologia de plantas, sua interpretação e representação ${ }^{1}$
}

\author{
CINARA S.C. BENCKE ${ }^{2,3}$ e L. PATRÍCIA C. MORELLATO ${ }^{2,4}$
}

(recebido: 14 de fevereiro de 2001; aceito: 15 de maio de 2002)

\begin{abstract}
Comparison of two methods of plant phenology estimation, their interpretation and representation). This work compared two methods of plant phenological evaluation along with their graphical representation. The study was conducted at the Núcleo Picinguaba, Ubatuba, São Paulo State ( $23^{\circ} 22^{\prime}$ S and $44^{\circ} 48^{\prime}$ W), Brazil, in three Atlantic forest types: coastal-plain, lowland, and premontane forest. Observations on flowering, fruiting, leaf fall and leaf flushing tree phenology were carried out monthly from November/1994 to April/1996. We compared i) the Fournier's percent index of intensity - the intensity of phenological events was estimated according to an interval scale varying from 0 to 4 , with a $25 \%$ interval between classes; and ii) index of activity or percent of individuals. The index of activity and the Fournier's percent index of intensity provided distinct and complementary information, optimizing the data collection and making the analysis and interpretation of phenological patterns more simple and straightforward. We suggest the combined use of the two methods in the estimation and representation of species-level phenological data, and the proper differentiation between peak of activity and peak of intensity.

RESUMO - (Comparação de dois métodos de avaliação da fenologia de plantas, sua interpretação e representação). Este trabalho compara dois métodos de avaliação fenológica e sua representação gráfica. O estudo foi desenvolvido no Núcleo Picinguaba, Ubatuba, Estado de São Paulo ( $23^{\circ} 22^{\prime}$ S e $44^{\circ} 48^{\prime}$ W), em três formações florestais: floresta de restinga, floresta de planície e floresta de encosta. A floração, frutificação, queda de folhas e brotamento foram estimados mensalmente para cada indivíduo arbóreo, durante 18 meses. Foram comparados: i) percentual de intensidade de Fournier ou índice de intensidade: escala intervalar de cinco categorias de 0 a 4 , com intervalo de $25 \%$ entre classes; e ii) índice de atividade ou porcentagem de indivíduos. O índice de atividade e o percentual de intensidade de Fournier forneceram informações distintas e complementares, facilitando a análise e interpretação do comportamento fenológico. Sugere-se que em estudos fenológicos populacionais sejam utilizados estes dois índices combinados para a análise e representação dos dados e que seja feita a distinção entre pico(s) de atividade e pico(s) de intensidade das fenofases.
\end{abstract}

Key words - Atlantic forest, phenology, methods, tropical forest, synchrony

\section{Introdução}

A heterogeneidade dos estudos que abordam direta ou indiretamente a fenologia de plantas resulta na falta de padronização dos termos e dos métodos adotados tanto para a coleta como para a análise dos dados (Frankie et al. 1974, Kharin 1976, Schirone et al. 1990, Newstrom et al. 1994a), causando imprecisão na descrição dos padrões encontrados e dificultando a interpretação e comparação dos resultados (Newstrom et al. 1994b).

Os ciclos fenológicos de plantas tropicais são complexos, apresentando padrões irregulares de difícil

\footnotetext{
1. Parte da dissertação de mestrado de C.S.C. Bencke.

2. Universidade Estadual Paulista, IB, Departamento de Botânica e Grupo de Fenologia de Plantas e Dispersão de Sementes, Caixa Postal 199, 13506-900 Rio Claro, SP, Brasil.

3. Universidade Federal do Rio Grande do Sul, Departamento de Ecologia, Caixa Postal 15007, 91540-000 Porto Alegre, RS, Brasil.

4. Autor para correspondência: pmorella@rc.unesp.br
}

reconhecimento, principalmente em estudos de curto prazo. Portanto, a escolha dos métodos de avaliação e representação tem especial importância, pois pode dificultar ou auxiliar no reconhecimento dos padrões fenológicos (Newstrom et al. 1994a). Estudos fenológicos em florestas tropicais envolvendo observação direta de plantas têm utilizado, em sua maioria, dois métodos de avaliação: 1. qualitativa, definindo apenas a pesença ou ausência da fenofase considerada e 2. semi-quantitativa, com a aplicação de categorias de quantificação que estimam a intensidade do evento fenológico em cada indivíduo, sendo mais comum a utilização de cinco categorias de quantificação (0 a 4), com intervalo de $25 \%$ entre elas (e.g. Bullock \& Solis-Magallanes 1990, Morellato et al. 1990, 2000, Smith-Ramirez \& Armesto 1994, Sun et al. 1996, Talora $\&$ Morellato 2000).

O presente estudo teve como objetivo comparar estes dois métodos de avaliação fenológica, descrevendo e analisando os padrões fenológicos observados de acordo com cada método. Também é 
apresentada uma forma de representação dos dados fenológicos que facilite a sua descrição, a comparação de padrões fenológicos distintos e o estabelecimento de uma relação com fatores ambientais.

\section{Material e métodos}

Este estudo foi desenvolvido no Núcleo de Desenvolvimento Picinguaba (NDP), município de Ubatuba $\left(23^{\circ} 22^{\prime}\right.$ S e $44^{\circ} 48^{\prime}$ W), litoral norte do Estado de São Paulo. O clima da região é tropical chuvoso, apresentando uma estação superúmida de outubro a abril, com precipitação média superior a $200 \mathrm{~mm}$ mensais, e uma estação menos úmida de maio a setembro, com precipitação média acima de $100 \mathrm{~mm}$ mensais (Morellato et al. 2000). A precipitação média anual é de $2.100 \mathrm{~mm}$ e a temperatura média anual é cerca de $22{ }^{\circ} \mathrm{C}$.

As observações foram conduzidas em três áreas de floresta do NDP: a "floresta de restinga", localizada na planície costeira, distante cerca de $500 \mathrm{~m}$ da beira da praia, a "floresta de planície", localizada na planície litorânea junto à base de uma encosta e distante cerca de $2,5 \mathrm{~km}$ da beira da praia, e a "floresta de encosta", localizada às margens do Rio da Fazenda, distante cerca de $4 \mathrm{~km}$ da beira da praia. Uma descrição detalhada das áreas amostradas é apresentada em Cesar \& Monteiro (1995), Sanchez et al. (1999) e Morellato et al. (2000).

Seleção das espécies amostradas - Foram selecionadas 14 espécies arbóreas do dossel e sub-dossel com circunferência mínima a altura do peito maior que $10 \mathrm{~cm}$ e boa visibilidade da copa e com número mínimo de cinco indivíduos amostrados por espécie (Fournier \& Charpantier 1975) em pelo menos uma das áreas. O número de indivíduos por espécie variou de 5 a 21, dependendo da abundância da espécie na floresta considerada. Para mais detalhes sobre a amostragem das espécies em cada área e sua fenologia ver Morellato et al. (2000) e Bencke \& Morellato (2002).

Coleta de dados - O período de observação foi de novembro de 1994 a maio de 1996. As observações foram mensais, registrando-se a presença das fenofases floração (antese), frutificação (frutos maduros), brotamento e queda foliar. A intensidade dos eventos fenológicos foi estimada para cada árvore seguindo-se os critérios de Fournier (1974) (ver Análise de dados). O material botânico coletado está depositado no Herbarium Rioclarense (HRCB) da Universidade Estadual Paulista, Instituto de Biociências de Rio Claro.

Análise de dados - Dois métodos de análise foram aplicados aos dados coletados, a fim de verificar que tipo de informação cada um deles revela sobre o comportamento fenológico das populações amostradas e como estas informações podem ser representadas graficamente (neste trabalho, o termo população é utilizado com o sentido de amostra populacional, uma vez que as florestas estudadas são bastante próximas entre si, de forma que os indivíduos amostrados para cada espécie possivelmente pertencem a uma única população). Os métodos aplicados foram:

Percentual de intensidade de Fournier - Neste método, proposto por Fournier (1974), os valores obtidos em campo através de uma escala intervalar semi-quantitativa de cinco categorias ( 0 a 4 ) e intervalo de $25 \%$ entre cada categoria, permitem estimar a porcentagem de intensidade da fenofase em cada indivíduo. Em cada mês, faz-se a soma dos valores de intensidade obtidos para todos os indivíduos de cada espécie e divide-se pelo valor máximo possível (número de indivíduos multiplicado por quatro). $\mathrm{O}$ valor obtido, que corresponde a uma proporção, é então multiplicado por 100, para transformá-lo em um valor percentual.

Índice de atividade (ou porcentagem de indivíduos) - Método mais simples, no qual é constatada somente a presença ou ausência da fenofase no indivíduo, não estimando intesidade ou quantidade. Esse método de análise tem caráter quantitativo em nível populacional, indicando a porcentagem de indivíduos da população que está manifestando determinado evento fenológico. Este método também estima a sincronia entre os indivíduos de uma população (Morellato et al. 1990), levando-se em conta que quanto maior o número de indivíduos manifestando a fenofase ao mesmo tempo, maior é a sincronia desta população. Foram apresentados apenas os resultados gráficos das espécies nas quais as diferenças e semelhanças entre os métodos são mais evidentes.

\section{Resultados}

Os métodos analisados neste estudo forneceram informações essencialmente diferentes (tabela 1). $\mathrm{O}$ índice de atividade revelou o período em que uma determinada fenofase ocorreu de maneira mais generalizada na população, permitindo visualizar melhor do que o índice de intensidade, nos gráficos, o início e o final dos períodos de atividade (figura 1). Esse índice forneceu, também, informações sobre sincronismo, indicando a proporção de indivíduos da população que estava manifestando simultaneamente um determinado evento fenológico. O percentual de intensidade de Fournier, por outro lado, apontou picos de intensidade, ou seja, mostrou quando uma determinada fenofase ocorreu de modo mais intenso na população, enfatizando a quantidade estimada de flores, frutos, folhas ou brotos produzidos e não apenas o número de indivíduos que manifestou a fenofase.

Os picos de atividade e de intensidade nem sempre coincidiram para as 14 espécies amostradas. Seis espécies (42,9\% do total) apresentaram essa assincronia, que foi mais freqüente, nessas espécies, nas fenofases vegetativas $(83,3 \%)$ do que nas reprodutivas $(16,7 \%)$. 
Tabela 1. Comparação dos dois métodos de avaliação dos dados fenológicos analisados no presente estudo.

Características
Índice de atividade

Índice de intensidade
Avaliação rápida das fenofases no campo

Permite identificar o início e o final dos períodos de atividade Indica sincronismo

Pode ser aplicado à análise de dados de herbário

Evidencia picos de intensidade

Quantificação da fenofase

$\begin{array}{cc}\text { Sim } & \text { Não } \\ \text { Sim } & \text { Sim } \\ \text { Sim } & \text { Não } \\ \text { Sim* } & \text { Não } \\ \text { Não } & \text { Sim } \\ \text { Objetiva } & \text { Subjetiva }\end{array}$

* Considera a presença/ausência do evento fenológico em cada exsicata examinada.

Por exemplo, em Lamanonia ternata Vell. o brotamento foi concentrado nos períodos entre novembro/1994 e junho/1995 e entre novembro/1995 e abril/1996, com picos de atividade em dezembro/1994, janeiro/1995, março/1995, dezembro/1995 e fevereiro/1996, enquanto que a intensidade máxima (pico de intensidade) desta fenofase ocorreu em novembro e dezembro/1994 e em dezembro/1995 (figura 1B). A falta de coincidência entre os picos de atividade e intensidade tende a ser mais evidente nas fases vegetativas porque nestas $o$ incremento cumulativo na magnitude da fenofase (em \% de indivíduos ou de intensidade) é mais lento e gradual
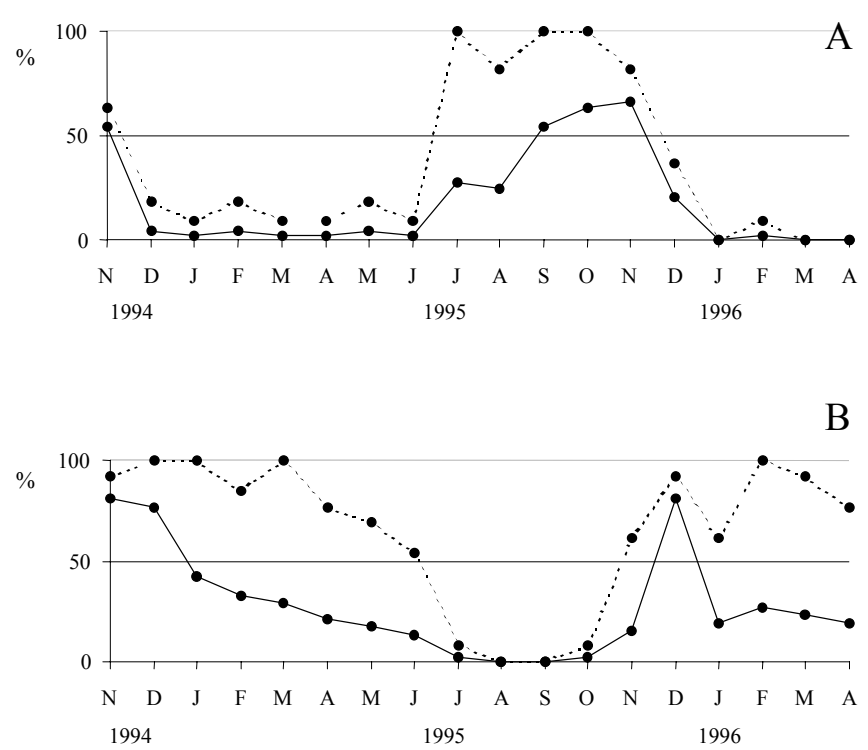

Figura 1. Representação da fenologia de acordo com dois métodos de análise de dados: índice de atividade (- - - -) e percentual de intensidade de Fournier (—• - para A. Queda foliar em Swartzia flaeminguii Raddi $(\mathrm{n}=11)$ e B. Brotamento em Lamanonia ternata Vell. ( $\mathrm{n}=13)$, amostradas na floresta de planície. do que nas fenofases reprodutivas, podendo ser detectado mesmo quando as observações de campo são separadas por intervalos de tempo relativamente longos (observações mensais, como no presente estudo). Portanto, o pico de atividade ocorre no momento em que o número máximo de indivíduos da amostra apresenta a fenofase, porém não necessariamente com a intensidade máxima, como por exemplo, quando muitos indivíduos manifestam baixa intensidade da fenofase (figura 1A, mês de julho/1995). Já o pico de intensidade ocorre no período em que os níveis de intensidade são mais elevados, mas a fenofase não é necessariamente manifestada por todos os indivíduos, ou seja, apenas alguns indivíduos apresentam a fenofase com maior intensidade. Da mesma forma, na primeira floração de Guatteria australis St. Hil. (figura 2A) o pico de intensidade ocorreu em dezembro-janeiro de 1994/1995 (índice de Fournier $=27,4 \%$ ), enquanto o pico de atividade ocorreu em dezembro, com cerca de $76 \%$ dos indivíduos floridos; em janeiro de 1994/1995 a porcentagem de indivíduos floridos (índice de atividade) caiu para $57 \%$, embora a porcentagem de intensidade continuasse a mesma do mês anterior. Já na segunda floração, a maior intensidade de floração foi em fevereiro/1996 (índice de Fournier $=19,1 \%$ ), embora a portentagem de indivíduos florindo tenha sido a mesma (48\%) de dezembro a fevereiro/1996 (figura 2A). Mesmo quando os picos de atividade e intensidade coincidem perfeitamente, sua interpretação pode ser distinta. Por exemplo, comparando as duas florações de Swartzia flaemingii Raddi (figura 2B) percebe-se que, embora o percentual de intensidade de Fournier tenha sido o mesmo nos dois anos (22\%), o índice de atividade (\% de indivíduos) foi maior em março/96 (81,5\%) do que em março/1995 (55\%).

A aplicação do índice de atividade e do percentual de intensidade de Fournier conjugados na avaliação e representação gráfica de dados fenológicos revelam 

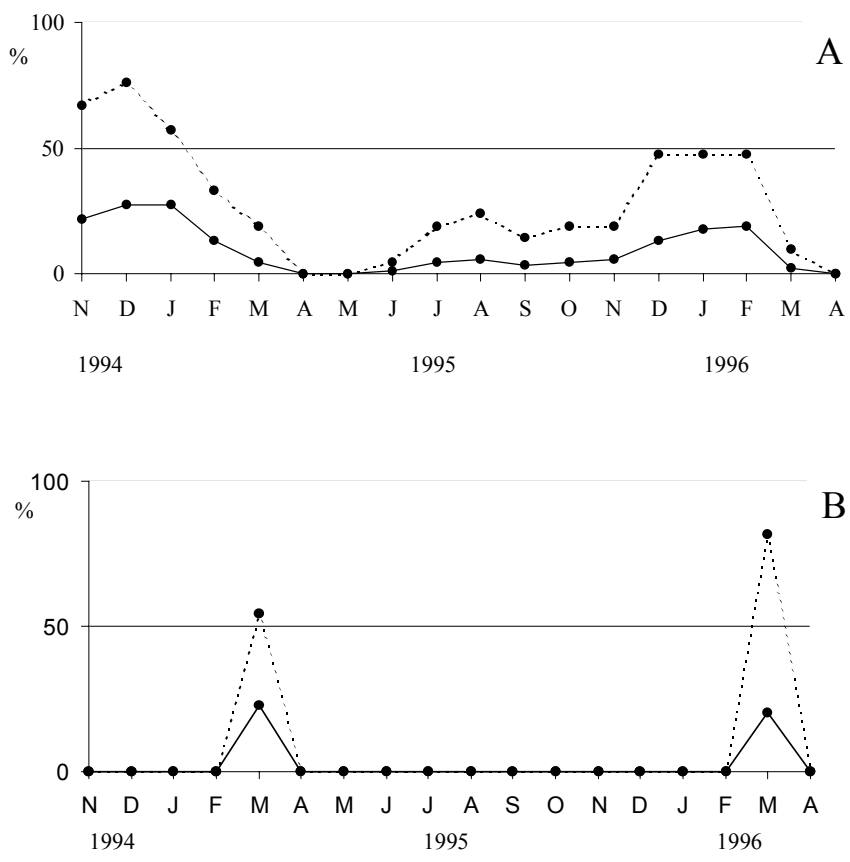

Figura 2. Representação da fenologia de acordo com dois métodos de análise de dados: índice de atividade (- - - -) e percentual de intensidade de Fournier ( - - ) para A. Antese em Guatteria australis St. Hil. $(\mathrm{n}=21)$ e B. Antese em Swartzia flaemingii Raddi $(\mathrm{n}=11)$, amostradas na floresta de planície.

mais informações e facilitam a descrição, comparação e análise dos padrões encontrados, como ilustra bem o exemplo na figura 3. Na floração, por exemplo, o índice de atividade permite identificar um período único de atividade na população, com três meses de duração e elevado sincronismo entre os indivíduos nos últimos dois meses. Já o percentual de intensidade de Fournier revela que esta fenofase foi intensa apenas no segundo mês de atividade, seguida por uma redução acentuada e abrupta na quantidade de flores no terceiro mês. $\mathrm{Na}$ frutificação, a atividade e o sincronismo entre os indivíduos foram menores do que na floração. A intensidade desta fenofase, ao contrário do que ocorreu na floração, aumentou gradualmente, atingindo o pico no segundo mês de atividade, o que se deveu provavelmente ao acúmulo de frutos maduros nos indivíduos. Quanto à queda foliar, o percentual de intensidade de Fournier mostrou um padrão predominantemente contínuo e homogêneo na intensidade da fenofase, enquanto o índice de atividade revelou que houve uma variação considerável no número de indivíduos que manifestaram esta fenofase ao longo das observações. Por fim, o gráfico que representa o brotamento mostra que o índice de atividade e o percentual de intensidade de Fournier podem apresentar comportamentos muito semelhantes.

A figura 3 é também um exemplo de apresentação de dados fenológicos, que associa a representação das fenofases por diferentes índices às variações de fatores ambientais no mesmo período, assim como à estacionalidade climática na área de estudo. O fenograma sugerido inclui: comprimento do dia na região (fator luz), a variação das temperaturas e a precipitação mensal no período de estudo. Note-se que a área sombreada destaca a estação superúmida, definida com base nos dados médios (30 anos) para a região. Dessa forma, a compreensão e descrição da fenologia e sua correspondência com fatores ambientais tornam-se mais direta, fácil e objetiva.

\section{Discussão}

Os métodos que vêm sendo utilizados em estudos fenológicos baseiam-se em dados obtidos através de escalas de mensuração em que são atribuídos valores numéricos aos diferentes níveis de atividade ou intensidade do ciclo biológico. Estas escalas de mensuração, embora variadas, podem ser classificadas essencialmente em nominais, quando indicando apenas presença/ausência (e.g., Alencar et al. 1979, Morellato et al. 1989, 1990), e intervalares semi-quantitativas, em que a razão entre os valores da escala seja conhecida (e.g. Morellato et al. 2000, Talora \& Morellato 2000, Heideman 1989). No presente trabalho essas escalas correspondem, respectivamente, ao índice de atividade e ao percentual de intensidade de Fournier (1974). Alguns autores utilizam ainda escalas ordinais, onde os valores ou "ranks" são atribuídos aos indivíduos observados, não havendo uma razão conhecida e constante entre eles. Por exemplo, a fenofase pode ser avaliada como sendo ausente, pouco intensa ou muito intensa (e.g., Frankie et al. 1974, Opler et al. 1980). Finalmente, alguns autores utilizam escalas mistas (e.g., Ortiz 1990, Borchert 1980). Especificamente para a quantificação dos eventos fenológicos reprodutivos (floração e frutificação) pode-se, ainda, adotar uma escala de mensuração quantitativa, por exemplo, quando são contadas todas as flores ou frutos de um indivíduo ou ramo (Araújo et al. 1994, Worthington 1996, Pombal \& Morellato 2000).

Os principais trabalhos de enfoque populacional já realizados no Brasil têm procedido à análise dos dados utilizando apenas um método, seja o índice de atividade (Morellato \& Leitão Filho 1990, Morellato et al. 1990, Costa et al. 1997) ou percentual de intensidade de Fournier (Talora \& Morellato 2000). Há ainda estudos cujas 

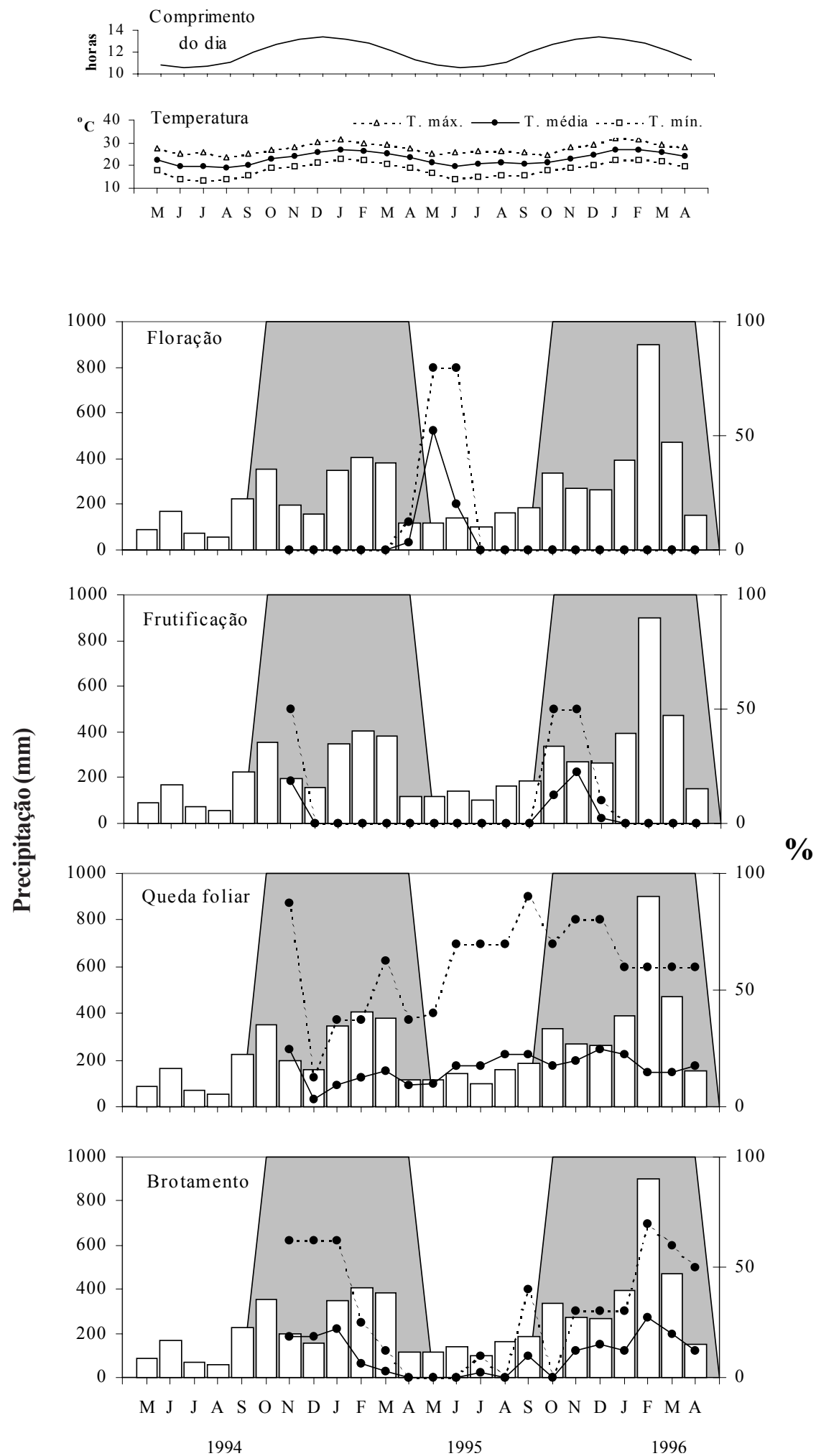

Figura 3. Exemplo de representação gráfica (fenograma) composta de dados climáticos e dados fenológicos de cada fenofase, para a espécie Cupania oblongifolia Mart. $(\mathrm{n}=10)$ de acordo com dois métodos de análise de dados: índice de atividade (-----) e percentual de intensidade de Fournier (-•-). Indivíduos amostrados na floresta de planície. Convenções: comprimento do dia na lat. $23^{\circ} \mathrm{S}$ (Ometto 1981); barras = precipitação total mensal do período de estudo; áreas em cinza = períodos superúmidos (médias de precipitação mensal dos últimos 30 anos acima de $200 \mathrm{~mm}$ ). 
observações se baseiam em escalas nominais (Alencar et al. 1979, Alencar 1994) ou intervalares (Morellato et al. 2000), mas não calculam os índices de intensidade e de Fournier, respectivamente.

A análise de dois diferentes métodos de avaliação dos dados fenológicos, realizada no presente estudo, indicou que estes fornecem informações distintas e complementares sobre o comportamento fenológico em amostras populacionais. A associação do período de atividade aos níveis de intensidade de uma fenofase, além de facilitar a análise e a interpretação do comportamento fenológico da espécie, pode representar um importante papel nas pesquisas sobre interações animal-planta, pois combina informações de sincronia e quantidade.

Heideman (1989) constatou que a proporção de indivíduos (índice de atividade) em floração ou frutificação nem sempre é um bom preditor do volume de flores ou frutos produzidos (i.e., da intensidade da fenofase), o que ressalta a importância de se conjugar os dois métodos, como sugerido no presente estudo, sobretudo para obter informações sobre a disponibilidade quantitativa e temporal deste tipo de recurso.

O desajuste temporal observado entre os picos de atividade e de intensidade salienta a importância de fazer-se a distinção entre eles, principalmente quando o nível de abordagem é populacional. O pico de intensidade da fenofase pode estar mais relacionado a fatores bióticos tais como a presença, atividade ou abundância de polinizadores e dispersores, uma vez que guarda relação direta com a abundância do recurso (flores ou frutos). O pico de atividade, por sua vez, pode estar mais relacionado com características endógenas e com fatores abióticos que atuam sobre a fisiologia da planta, determinando ou restringindo o período de ocorrência das fenofases.

Em estudos de comunidades nos quais um grande número de indivíduos de várias espécies precisa ser periodicamente monitorado, a aplicação de uma escala nominal como o índice de atividade torna mais ágil a coleta de dados. O percentual de intensidade de Fournier, por outro lado, exige tempo e experiência do observador, entretanto mostra os picos e tendências da fenofase, distinguindo vários níveis de intensidade. Este tipo de detalhamento e refinamento das informações fenológicas é desejável especialmente em estudos populacionais e análises de correlação, como mostra o estudo de Bencke \& Morellato (2002). Sugerimos ainda que, em estudos de fenologia, seja feita a distinção entre "pico de atividade" e "pico de intensidade", conforme o método utilizado, ao invés de "pico de fenofase", tornando a descrição e interpretação dos dados fenológicos mais objetiva e precisa.

Agradecimentos - As autoras agradecem a F. F. d'Eça Neves, F.A.M dos Santos, V.B. Zipparro, G. A. Bencke e a dois revisores anônimos pelas sugestões e críticas ao manuscrito; ao Instituto Florestal pela permissão de pesquisa no Núcleo Picinguaba e aos funcionários pelo auxílio nos trabalhos de campo; à Fapesp (proc. n. 93/3604-9) pelo apoio financeiro; ao CNPq pela bolsa de mestrado de C.S.C.B. e de Produtividade em Pesquisa de L.P.C.M. A análise dos dados e redação deste trabalho foi facilitada pelo apoio da Fapesp ao Laboratório de Banco de Dados Fenológicos (proc. n. 96/9626-0).

\section{Referências bibliográficas}

ALENCAR, J.C. 1994. Fenologia de cinco espécies arbóreas tropicais de Sapotaceae correlacionada a variáveis climáticas na reserva Ducke, Manaus, AM. Acta Amazonica 24:161-182.

ALENCAR, J.C., ALMEIDA, R.A. \& FERNANDES, N.P. 1979. Fenologia de espécies florestais em floresta tropical úmida de terra firme na Amazônia Central. Acta Amazonica 9:163-198.

ARAUJO, A.C., FISCHER, E.A. \& SAZIMA, M. 1994. Floração sequencial e polinização de três espécies de Vriesea (Bromeliaceae) na região de Juréia, sudeste do Brasil. Revista Brasileira de Botânica 17:113-118.

BENCKE, C.S.C. \& MORELLATO, L.P.C. 2002. Estudo comparativo da fenologia de nove espécies arbóreas em três tipos de floresta atlântica no sudeste do Brasil. Revista Brasileira de Botânica 25:237-248.

BULLOCK, S.H. \& SOLIS-MAGALLANES, J.A. 1990. Phenology of canopy trees of a tropical deciduous forest in Mexico. Biotropica 22:22-35.

BORCHERT, R. 1980. Phenology and ecophysiology of tropical trees: Erythryna poeppigiana O.F. Cook. Ecology 61:1065-1074.

CESAR, O. \& MONTEIRO, R. 1995. Florística e fitossociologia de uma floresta de restinga em Picinguaba (Parque Estadual da Serra do Mar), Município de Ubatuba, SP. Naturalia 20:89-105.

COSTA, M.L.M.N, ANDRADE, A.C.S. \& PEREIRA, T.S. 1997. Fenologia de espécies em floresta montana na Reserva Ecológica de Macaé de Cima. In Serra de Macaé de Cima: diversidade florística e conservação em Mata Atlântica. (H.C. Lima \& R.R. Guedes-Bruni, eds.). Instituto de Pesquisas Jardim Botânico do Rio de Janeiro, Rio de Janeiro, p.169-186.

FOURNIER, L.A. 1974. Un método cuantitativo para la medición de características fenológicas en árboles. Turrialba 24:422-423. 
FOURNIER, L.A. \& CHARPANTIER, C. 1975. El tamaño de la muestra y la frecuencia de las observaciones en el estudio de las características fenológicas de los árboles tropicales. Turrialba 25:45-48.

FRANKIE, G.W, BAKER, H.G. \& OPLER, P.A. 1974. Comparative phenological studies of trees in tropical wet and dry forests in the lowlands of Costa Rica. Journal of Ecology 62:881-913.

HEIDEMAN, P.D. 1989. Temporal and spatial variation in the phenology of flowering and fruiting in a tropical rainforest. Journal of Tropical Ecology 77:1059-1079.

KHARIN, N.G. 1976. Mathematical models in phenology. Journal of Biogeography 3:357-364.

MORELLATO, L.P.C. \& LEITÃO FILHO, H.F. 1990. Estratégias fenológicas de espécies arbóreas em floresta mesófila na Serra do Japi, Jundiaí, São Paulo. Revista Brasileira de Biologia 50:163-173.

MORELLATO, L.P.C., RODRIGUES, R.R., LEITÃO FILHO, H.F. \& JOLY, C.A. 1989. Estudo comparativo da fenologia de espécies arbóreas de floresta de altitude e floresta mesófila semi-decídua na Serra do Japí, Jundiaí, São Paulo. Revista Brasileira de Botânica 12:85-98.

MORELLATO, L.P.C., LEITÃO FILHO, H.F., RODRIGUES, R.R. \& JOLY, C.A. 1990. Estratégias fenológicas de espécies arbóreas em floresta de altitude na Serra do Japi, Jundiaí São Paulo. Revista Brasileira de Biologia 50:149-162.

MORELLATO, L.P.C., TALORA, D.C., TAKAHASI, A., BENCKE, C.S.C., ROMERA, E.C. \& ZIPPARRO, V. 2000. Phenology of atlantic rain forest trees: a comparative study. Biotropica 32(Special Issue): 811-823.

NEWSTROM, L.E., FRANKIE, G.W. \& BAKER, H.G. 1994a. A new classification for plant phenology based on flowering patterns in lowland tropical rain forest trees at La Selva, Costa Rica. Biotropica 26:141-159.

NEWSTROM, L.E., FRANKIE, G.W., BAKER, H.G. \& COLWELL, R.K. 1994b. Diversity of long-term flowering patterns. In La Selva: Ecology and natural history of a neotropical rain forest (L.A. McDade, K.S. Bawa, H.A. Hespenheide \& G.S. Harstshorn, eds.). University of Chicago Press, Chicago, p.142-160.
OMETTO, J.C. 1981. Bioclimatologia vegetal. Agronomica Ceres, Piracicaba.

OPLER, P.A., FRANKIE, G.W. \& BAKER, H.G. 1980. Comparative phenological studies of treelet and shrub species in tropical wet and dry forests in the lowlands of Costa Rica. Journal of Ecology 68:167-188.

ORTIZ, R. 1990. Fenologia de árboles en un bosque semideciduo tropical del estado Cojedes. Acta Botanica Venezuelana 16:93-116.

POMBAL, E.C.P. \& MORELLATO, L.P.C. 2000. Differentiation of floral color and odor in two fly pollinated species of Metrodorea (Rutaceae) from Brazil. Plant Systematics and Evolution 221:141-156.

SANCHEZ, M., PEDRONI, F., LEITÃO FILHO, H.F. \& CESAR, O. 1999. Composição florística de um trecho de floresta ripária na Mata Atlântica em Picinguaba, Ubatuba, SP. Revista Brasileira de Botânica 22:31-42.

SCHIRONE, B., LEONE, A., MAZZOLENI, S. \& SPADA, F. 1990. A new method of survey and data analysis in phenology. Journal of Vegetation Sciences 2:27-34.

SMITH-RAMIREZ, C. \& ARMESTO, J.J. 1994. Flowering and frutiting patterns in the temperate rainforest of Chiloé, Chile: ecologies and climatic constraints. Journal of Ecology 82:353-365

SUN, C., KAPLIN, B.A., KRISTENSEN, K.A., MUNYALIOGA， V., MVUKIYUMWAMI，J., KAJONDO, K.K. \& MOERMOND, T.C. 1996. Tree phenology in a tropical montane forest in Rwanda. Biotropica 28:668-681.

TALORA, D.C. \& MORELLATO, L.P.C. 2000. Fenologia de espécies arbóreas em floresta de planície litorânea do sudeste do Brasil. Revista Brasileira de Botânica 23:13-26.

WORTHINGTON, A. 1996. Population sizes and breeding rhythms of two species of manakins in relation to food supply. In The ecology of a tropical forest: Seasonal rhythms and long-term changes (E. Leigh Jr., A.S. Rand \& D. Windsor, eds.). Smithsonian Institution Press, Washington, p.213-225. 